\title{
LOS BLOGS COMO RECURSO DE LA ORIENTACIÓN PROFESIONAL EN LA WEB 2.0
}

\section{BLOGS AS A RESOURCE FOR CAREER GUIDANCE IN WEB 2.0}

\author{
Pilar Martínez-Clares \\ Mirian Martínez-Juárez \\ Francisco Javier Pérez Cusó \\ Universidad de Murcia. Facultad de Educación. Departamento de Métodos de Investigación y \\ Diagnóstico en Educación. Murcia, España
}

\section{RESUMEN}

Las TIC se presentan como un importante recurso al servicio de los profesionales de la orientación profesional, favoreciendo la comunicación, el trabajo colaborativo o la interacción con los usuarios, entre otros aspectos. Los blogs son una herramienta privilegiada en este sentido, ya que presentan como característica propia facilitar la construcción de conexiones sociales significativas alrededor de cualquier tema. Por ello, desde este trabajo se plantea como propósito principal profundizar en la blogosfera de la orientación profesional, la actividad de publicación en la misma, la visibilidad de los blogs, la comunidad generada en torno a éstos y las interacciones que se producen en torno a los mismos. Para ello se recoge información de un total de 136 blogs de orientación profesional y se analizan 4516 publicaciones (posts), recogiendo diferentes variables como tipología de contenido e interacción que se produce en torno a las mismas. Los resultados muestran una importante heterogeneidad en los blogs en cuanto a sus autores y contenidos publicados. También hay diferencias en relación a la comunidad de usuarios e interacciones que se generan. En relación a la visibilidad depende en gran medida de la tipología de contenido publicado, aunque esta resulta siempre escasa. Todo esto, inicialmente, permite concluir que parece no existir un modo unívoco

\footnotetext{
${ }^{1}$ Correspondencia: Mirian Martínez Juárez. Facultad de Educación. Campus Universitario de Espinardo. 30100 Murcia (España). Correo-e: mmartinez@um.es
} 
de entender y trabajar en la blogosfera de la orientación profesional, por lo que sus posibilidades como herramienta o recurso son amplias. Se subraya que no es suficiente una conexión unidireccional, sino que se debe profundizar en elementos clave del proceso orientador como la comunicación, vinculación o confianza.

Palabras clave: Orientación profesional, redes sociales (en línea), internet, TIC, análisis de contenido.

\begin{abstract}
Information and Communication Technologies (ICT) are an important tool for career guidance professionals. These tools, and blogs as one of the most important, can favor communication, collaborative work, and interaction with users, among many other elements. Blogs are a privileged tool in this sense because they facilitate the construction of meaningful social connections around any topic. This research aims to improve knowledge about the career guidance blogosphere, the publication activity in it, the visibility of blogs on the web, the community generated around them and the interactions that occur in relation to the published content. To do this, information from 136 career guidance blogs and 4516 publications (posts) was collected and analyzed, including different variables such as content type and interaction around them. The results show an important heterogeneity in the blogs, their authors, contents, visibility, as well as the relation to the community and interactions that are generated. Visibility, always low, depends largely on the type of content published. We conclude that there seems not to be a unique way of understanding and working on career guidance in the blogosphere, which shows the breadth of its possibilities as a resource. It is emphasized that a one-way connection is not enough, highlighting some key elements of career guidance, such as interaction, bonding, or trust.
\end{abstract}

Keywords: Career guidance, social networks (online), internet, ICT, content analysis.

\title{
Cómo citar este artículo:
}

Martínez-Clares, P., Martínez-Juárez, M. y Pérez Cusó, F.J. (2020). Los blogs como recurso de la orientación profesional en la web 2.0. Revista Española de Orientación y Psicopedagogía, 31(3), 725. https://doi.org/10.5944/reop.vol.31.num.3.2020.29259

\section{Introducción}

Vivimos enredados o, al menos, ese es el eslogan que repiten numerosos autores cuando pretenden describir la sociedad de hoy en día. Con este juego de palabras se intenta caracterizar nuestra sociedad, una sociedad en red, organizada a partir de numerosos nodos interconectados, constituidos por diferentes entidades, organismos, instituciones $\mathrm{y}$, hoy más que nunca, por las 
personas consideradas individualmente, independientemente de que estas puedan constituirse en grupos más o menos formales (Castells, 2012).

En esta estructura de la sociedad del siglo XXI, las Tecnologías de la Información y la Comunicación (TIC, en adelante) se constituyen como medios esenciales; concretamente, el avance de la microelectrónica, la informática y las telecomunicaciones traen como consecuencia lo que se denomina cuarta revolución industrial (Schwab, 2016); para otros no es posible determinar su capacidad de irrupción, es decir, hasta qué punto será revolución como la máquina de vapor en la primera, la producción en cadena en la segunda o el ordenador en la tercera (De Pablos, 2016).

De momento, el nivel de digitalización que se está imprimiendo en todos los ámbitos y sectores de la sociedad no tiene precedentes, lo que también es posible gracias a la evolución constante de Internet. En contraposición a sus inicios, sus posibilidades hoy en día son numerosas, de tal manera que "se concibe no sólo como un medio de comunicación..., sino como un espacio social de interacción" (Gómez Cruz, 2002, p. 276-277), en el que es posible una "constelación de vínculos relacionales" entre personas, lo que a su vez permite multitud de "interacciones sociales en el ciberespacio, un espacio social sin lugar físico" (Del Fresno, Daly y Segado, 2016, p. 24).

En la web 2.0, el usuario no sólo comparte información, sino que también la crea, comenta e interactúa con ella, asumiendo así nuevos roles en el proceso de comunicación digital (Fernández González, 2015; Piñeiro, 2019). De esta manera, el intercambio dinámico se convierte en la principal característica de la web 2.0. Con su consolidación se dibuja un nuevo entorno, en el que los usuarios encuentran un amplio abanico de recursos, de tal manera que en ocasiones no sólo se habla de usuarios visitantes, es decir, aquellas personas que se conectan a ese espacio virtual puntualmente para desarrollar tareas concretas (revisar correo, buscar información, interactuar con un contacto o leer noticias), sino de usuarios residentes, que se caracterizan por no diferenciar lo que hacen en el mundo on-line y en el mundo off-line (Hernández, Ramírez-Martinell y Cassany, 2014).

Ante esta tendencia, la orientación profesional debe contemplar el ciberespacio y analizar, describir, comprender e integrar las posibilidades que ofrece a los usuarios, con la finalidad de aprovechar la oportunidad que brinda el mundo on-line para crear sinergias y construir comunidades virtuales de orientación. Para Cogoi (2002, p. 5), "la utilización de las TIC es cada vez más necesaria y urgente para enfrentarse a los cambios de una sociedad sin fronteras". Esto implica repensar el proceso orientador desarrollado hasta el momento para incluir nuevos medios, herramientas o recursos que permitan incrementar sus beneficios, dado que la orientación profesional tiene un gran papel, y asume un gran reto, a la hora de "preparar para un futuro imprevisible, donde las coordenadas, hasta hace poco asumidas y vigentes, muestran una cierta insuficiencia explicativa y predictiva" (Manzanares y Sanz, 2018, p.65).

Esta tendencia de crear comunidades virtuales de orientación puede variar, dependiendo del nivel de interacción y compromiso, hablándose de comunidades virtuales de interés, de aprendizaje o de práctica. Lo ideal sería llegar a consolidar estas últimas, las que no sólo presentan un mayor compromiso y colaboración, sino que trascienden el propio aprendizaje, tendiendo a la innovación y mejora continua (Suárez, Pérez, Boza y García-Valcárcel, 2012).

No obstante, como resalta Fuchs (2011), independientemente del carácter de la comunidad virtual que se genere, la web 2.0 o web social se caracteriza por constituir una comunidad de usuarios activos, lo que se evidencia en el uso de distintos medios como es el caso de las redes sociales, definidas como espacios de conexión constituidos en torno a diferentes intereses en los que prima el contacto, la comunicación, la interacción y el intercambio entre usuarios, lo que posibilita o da lugar a la información, al conocimiento, al aprendizaje y, por supuesto, a la orientación.

Como ocurre con las comunidades virtuales, también existen distintos tipos de redes sociales virtuales. Se habla de redes sociales indirectas y directas, según el Observatorio Nacional de las 
Telecomunicaciones y de la Sociedad de la Información (ONTSI, 2011). En el caso de las primeras existe un individuo o grupo que controla y dirige la información o las discusiones en torno a un tema concreto, como es el caso de los foros o los blogs. Por el contrario, las directas permiten una colaboración entre grupos de personas que comparten intereses y que, interactuando entre sí en igualdad de condiciones, pueden controlar la información que comparten. Los usuarios de este tipo de redes sociales crean perfiles a través de los cuales gestionan su información personal y la relación con otros usuarios, como es el caso de Twitter y Linkedln.

Las redes sociales indirectas son las precursoras de las redes sociales directas desarrolladas dentro del nuevo marco de la web 2.0. Entre ellas se encuentran los blogs, cuya presencia es tan fuerte en Internet que ha dado lugar a la blogosfera, en la que subyace un profundo fenómeno de comunicación.

La blogosfera es un espacio extenso, abierto, sin límites definidos y en proceso de construcción permanente, en el que se establecen comunidades virtuales en torno a distintas temáticas (Barraza, 2013). Definida de modo más gráfico, se trata de una colmena en la que habitan multitud de usuarios agrupados según sus intereses, conformando la estructura de un panal.

A partir de esta fundamentación, se deriva que los blogs presentan una serie de características propias relacionadas con facilitar la construcción de conexiones sociales significativas o comunidades virtuales alrededor de cualquier tema de interés (Barraza, 2013). Se definen como "una jerarquía de texto, imágenes, objetos multimedia y datos, ordenados cronológicamente, soportados por un sistema de distribución de contenidos capaz de proporcionar (al autor) la funcionalidad necesaria para distribuir esos contenidos con cierta frecuencia, exigiéndole unas capacidades técnicas mínimas" (Fumero, 2005, p. 49). Se trata de un medio centralizado, pues su actividad depende de su autor, y jerárquico, ya que éste es el único con privilegios para publicar contenidos (posts) (Orihuela, 2006), a partir de ahí se pretende generar una comunidad desde dentro hacia fuera: el autor propone los temas, establece la agenda y los usuarios responden, iniciándose así un proceso de intercambio, reflexión, relación y, por qué no, orientación.

Precisamente, pensando en su aplicabilidad a la orientación, de la misma manera que Sáez Vacas (2005) lo hace para la educación, la estructura hipertextual de sus entradas, publicaciones o posts (es decir, su estructura no lineal, en la que se insertan distintos hipervínculos o hiperenlaces que permiten la navegación y, en consecuencia, la profundización en la temática tratada a partir de otros recursos como una página web y/u otro blog), unida a la inmediatez, actualización y al poder de comunicación e intercambio de los blogs, hacen que se conviertan en recursos o herramientas para llevar a cabo la orientación en red, digital, virtual o la e-orientación.

Los hipervínculos, si se usan con precisión y mesura, confieren profundidad informativa y hasta epistemológica a un blog (Sáez Vacas, 2005). Así, un orientador puede crear un blog y realizar orientación, en forma de guía o acompañamiento a los usuarios o internautas (lectores de su blog) a través de un proceso interactivo hacia su desarrollo profesional, creando una comunidad virtual de orientación en cualquier rincón de la blogosfera, entendiendo que la orientación es un proceso que va más allá de una conversación, una consulta puntual o de la mera información. Siguiendo a Martínez Clares (2017), la orientación es un proceso global centrado en la persona, por lo que debe partir del conocimiento del orientado en cuanto a sus fortalezas, debilidades, intereses, expectativas, motivaciones, entre otros aspectos, y, por supuesto, del conocimiento de su entorno. Esto le llevará a una toma de decisiones adaptada y ajustada a su realidad, es decir, al esclarecimiento de posibilidades con sentido (Echeverría y Martínez, 2018). La finalidad última de la orientación es propiciar la autonomía de la persona a la hora de afrontar diferentes situaciones a lo largo de su vida, de ahí su carácter de acompañamiento y la necesidad de hacerlo actualmente, en la sociedad digital que vivimos, de forma virtual.

En cualquier caso, se hace necesario el desarrollo de una intervención continuada, es decir, un proceso que sitúa como protagonista de la acción al orientado, en el que se profundiza, se motiva, 
se informa, se asesora, se coordina, se media, se forma, en definitiva, se orienta. Un proceso en el que la persona acaba adquiriendo, desarrollando y demostrando una serie de competencias profesionales que le permitirán la consecución de sus metas y ser realmente quien es.

Ante esta panorámica, es interesante indagar y contestar a si es posible implementar este proceso a través de un blog de orientación, si realmente se hace, quién lo hace, cómo lo hace y si existe una comunidad virtual real y activa en relación a la orientación profesional generadora de conocimiento, intercambio, colaboración y aprendizaje o tan sólo un conjunto de blogs relacionados por su temática desde el que cada bloguero intenta mantener a sus seguidores y atraer otros nuevos.

Hasta el momento ya se ha intentado dar respuesta a algunos de estos interrogantes, aunque la e-orientación profesional como escenario de investigación es relativamente reciente (Martínez, González y Pérez, 2018). Como se constata en este último trabajo, diferentes son las iniciativas que ponen de relieve la importancia de las TIC para desarrollar procesos de orientación profesional eficaces (Fernández González, 2015; Sobrado, Ceinos y García, 2012), aunque no se han encontrado estudios que puedan servir de referencia directa, en los que se profundice en la blogosfera como generadora de recursos de orientación profesional para los usuarios y como espacio de colaboración e intercambio para los orientadores, ya que en el ámbito de la orientación es más frecuente encontrar el estudio de los blogs asociado a experiencias de innovación educativa (Cuevas, 2011; González-Benito y Otero, 2019; Muñoz-Carril y González-Sanmamed, 2014; Romero y Montilla, 2015; Veiga, Valverde, Castilla y Diz, 2017).

De ahí la decisión de iniciar una investigación que, en un primer momento, indague en la colmena de la blogosfera de la orientación profesional y, con este trabajo, en la cantidad, calidad y profundidad de las interacciones generadas por blogueros y usuarios, por lo que los objetivos establecidos se concretan en los siguientes: 1) conocer la actividad de publicación de los blogs seleccionados y relacionados con la orientación profesional, así como su visibilidad o posicionamiento en la red e 2) identificar la comunidad virtual generada en torno a dichos blogs, a través de diferentes tasas de interacción formuladas a partir de los debates iniciados con los contenidos más difundidos.

\section{Método}

Este trabajo se enmarca en una investigación más amplia cuya finalidad es indagar en la aportación de la Web 2.0 a la orientación profesional, con la intención de potenciar los beneficios del proceso orientador. Tiene un enfoque metodológico mixto y un diseño explicativo secuencial. El presente trabajo se centra en la primera etapa de dicha investigación, llevada a cabo desde un enfoque cuantitativo y con un diseño metodológico no experimental, descriptivo y transversal. En ella, desarrollada a lo largo del segundo semestre de 2018, se siguen los pasos que conforman la técnica de análisis de contenido cuantitativo (Hernández, Fernández y Baptista, 2014).

Así, en primer lugar, se define el universo a analizar para la extracción de una muestra representativa, constituido por los blogs en español relacionados específicamente con la orientación profesional. Su búsqueda se lleva a cabo a través de motores de búsqueda como Google y Bing (por tratarse de los dos más utilizados en el mundo según los datos proporcionados por Net Market Share para el segundo semestre de 2018), utilizando los descriptores "orientación+profesional", "orientación+laboral" y "orientación+empleo". También se aprovecha el Blogroll, elemento de la estructura de un blog que permite visualizar enlaces a otros blogs que el autor recomienda. La 
búsqueda termina una vez alcanzado el punto de saturación de la información, es decir, cuando los blogs encontrados no muestran nuevos contenidos y servicios y el Blogroll remite a otros ya registrados. Una vez realizada la búsqueda $(\mathrm{N}=221)$ se procede a efectuar la selección, optando por dos criterios: destinatarios del blog (de interés no exclusivo para usuarios del ámbito educativo formal o profesionales de recursos humanos) y frecuencia de publicación o nivel de actividad (activos en el momento de la selección, con al menos un post en los últimos 30 días). De esta forma la muestra queda constituida por 136 blogs, divididos en personales $(n=102)$ y corporativos $(n=34)$ y con una media de antigüedad de 5.14 (D.T.=2.60) y 4.35 años, respectivamente (D.T.=2.29).

En segundo lugar, se identifican las unidades de análisis o se concretan los elementos a explorar para dar respuesta a los objetivos de este trabajo. Uno de ellos es el perfil de los autores de los blogs que constituyen la muestra participante (sexo, edad, formación inicial, formación complementaria, ocupación actual y número de seguidores en distintas redes sociales: Twitter, Facebook, Linkedln). Para ello se tiene en cuenta la información del autor contenida en su blog. Señalar que el $66.3 \%$ de los blogueros son hombres mientras que el $33.7 \%$ son mujeres. Su edad media se encuentra en 43.20 (D.T.=7.39). Además, se recogen dos variables aportadas por Alexa para cada blog, una herramienta de análisis web que elabora un ranking de visibilidad de páginas web y ofrece el número de enlaces que direccionan a una web.

Otra unidad de análisis identificada son los posts publicados a lo largo del segundo semestre de 2018, que constituyen un total de 4516 entradas. A partir de estos se pueden deducir los contenidos abordados por cada bloguero durante dicho periodo. Junto a cada post también se recoge el número y tipo de comentarios generados, así como el número de veces compartido y/o de "me gusta" otorgados y la red social en la que se ha hecho. Con esta información, conjugada con el número de seguidores de cada blog en las redes sociales, se configuran nuevas variables que denominamos comunidad (1) y total de interacciones (2), como se aprecia en las siguientes fórmulas:

Comunidad $=$ Seguidores en LinkedIn + Seguidores en Facebook + Seguidores en Twitter

Total de interacciones $=$ Comentarios en el blog + Me gusta LinkedIN + Me gusta Twitter + Me gusta Facebook + Retweets

A partir de ahí, se generan diferentes tasas relativas a la interacción global (3), interacción de comentarios (4), interacción de Linkedln (5), de Facebook (6) y de Twitter (7), que responden a las siguientes fórmulas:

$$
\begin{gathered}
\text { Tasa de interacción global }=\frac{\text { Total de interacciones }}{\text { Comunidad }} \\
\text { Tasa de interacción (Comentarios) }=\frac{n^{\mathrm{o}} \text { comentarios en blog }}{\text { Comunidad }} \\
\text { Tasa de interacción (LinkedIn) }=\frac{n^{\mathrm{o} \text { compartidos LinkedIn }}}{\text { Comunidad }} \\
\text { Tasa de interacción }\left(\text { Facebook) }=\frac{n^{\mathrm{o} \text { compartidos Facebook }}}{\text { Comunidad }}\right. \\
\text { Tasa de interacción }(\text { Twitter })=\frac{n^{\mathrm{o} \text { Retwets }}}{\text { Comunidad }}
\end{gathered}
$$

Así, pese a que la audiencia de un blog ha de ser necesariamente más amplia, definimos comunidad como la suma de los seguidores de cada blog en su espacio o perfil respectivo en las 
tres redes sociales analizadas, asumiendo que es este segmento de la audiencia del blog el que mantiene una mayor vinculación con el mismo, su autor y los contenidos que publica.

La información es recogida a través de una ficha elaborada al efecto para el análisis de los blogs, a partir de las unidades de análisis identificadas y descritas anteriormente.

En tercer lugar, se establecen y definen las categorías y subcategorías que permiten la clasificación de la información recabada y representan las variables de la investigación. Como resultado se obtienen distintas categorías, como se verá a continuación, para codificar la formación y ocupación de los blogueros, así como los contenidos abordados a través de las distintas publicaciones, entradas o posts y los debates iniciados a partir de estos últimos. Por último, una vez realizada la codificación, se obtienen los totales para cada una de las categorías y se lleva a cabo el análisis de datos cuantitativo a través del programa estadístico SPSS v.24, empleando estadísticos descriptivos y pruebas no paramétricas para los análisis inferenciales, tomando como referencia el valor $p \leq .05$ para la detección de diferencias significativas.

\section{Resultados}

Objetivo1. Conocer la actividad de publicación de los blogs seleccionados y relacionados con la orientación profesional, así como su visibilidad o posicionamiento en la red.

Se realiza un análisis del nivel de actividad de los blogs, en el que se valora la frecuencia media mensual de publicación y se compara la frecuencia de publicación de los blogs de carácter personal y los institucionales. El análisis muestra diferencias significativas (U de Mann Whitney=1278.00, $p=.026$ ) entre ambas tipologías de blogs, siendo los institucionales los que publican con mayor frecuencia mensual $(\bar{X}=17.83$, D.T. $=43.34)$ frente a los personales $(\bar{X}=8.84$, D.T. $=34.29)$, tal vez con la idea de atraer más usuarios a su comunidad y realizar así una mayor publicidad de sus servicios, dado el carácter corporativo y de negocio de los blogs institucionales. En ambos casos, se observa una elevada desviación típica.

En un análisis más pormenorizado no se encuentran diferencias en el nivel de actividad según la formación de postgrado de los blogueros; en cambio, sí aparecen en la actividad promedio de los mismos según su ocupación y formación inicial universitaria, tal y como se muestra en la tabla 1.

\section{Tabla 1}

Promedio de publicaciones mensuales por ocupación y formación inicial del bloguero

\begin{tabular}{|c|c|c|c|c|c|c|}
\hline & & $\mathbf{N}$ & $\begin{array}{c}\text { Rango } \\
\text { promedio }\end{array}$ & $\overline{\mathbf{x}}$ & $\begin{array}{l}\text { Desviación } \\
\text { típica }\end{array}$ & $\begin{array}{c}x^{2} \\
\text { (gl) Sig. }\end{array}$ \\
\hline \multirow{5}{*}{ Ocupación } & Directores y gerentes & 15 & 40.07 & 3.60 & 7.70 & \multirow{5}{*}{$\begin{array}{c}9.593 \\
(4) \\
.048\end{array}$} \\
\hline & Consultor / Técnico RRHH & 31 & 51.87 & 2.93 & 3.90 & \\
\hline & Formador / docente & 13 & 48.27 & 2.03 & 1.40 & \\
\hline & Orientador & 34 & 54.82 & 20.22 & 58.62 & \\
\hline & Otros & 13 & 74.65 & 8.77 & 14.52 & \\
\hline
\end{tabular}

Fuente: Elaboración propia 
Tabla 1

Promedio de publicaciones mensuales por ocupación y formación inicial del bloguero

\begin{tabular}{|c|c|c|c|c|c|c|}
\hline & & $\mathbf{N}$ & $\begin{array}{c}\text { Rango } \\
\text { promedio }\end{array}$ & $\overline{\mathbf{x}}$ & $\begin{array}{c}\text { Desviación } \\
\text { típica }\end{array}$ & $\begin{array}{c}x^{2} \\
\text { (gl) Sig. }\end{array}$ \\
\hline \multirow[t]{5}{*}{ Formación } & ADE & 17 & 49.32 & 5.67 & 8.97 & \multirow{5}{*}{$\begin{array}{c}12.938 \\
(4) \\
.024\end{array}$} \\
\hline & Derecho y Ciencias del Trabajo & 23 & 51.11 & 12.23 & 34.07 & \\
\hline & Pedagogía, Psicología y Educación & 28 & 33.46 & 1.31 & 1.08 & \\
\hline & Sociología, Filosofía, Publicidad & 8 & 51.13 & 3.46 & 3.53 & \\
\hline & Titulaciones técnicas & 15 & 56.70 & 3.09 & 2.18 & \\
\hline
\end{tabular}

Fuente: Elaboración propia

En la tabla 1 se observan diferencias significativas en el nivel de actividad según la ocupación y formación inicial del bloguero, lo que pone de relieve el impacto del perfil de los autores en relación a su frecuencia de publicación. En ambos casos se relaciona directamente con la orientación, ya que la ocupación de los blogueros que más publican es la de orientador $(\mathrm{N}=34)$ y la formación de base hace referencia a Pedagogía, Psicología y titulaciones relacionadas con la Educación ( $\mathrm{N}=28$ ).

Para el análisis de visibilidad de los blogs se toman en cuenta dos variables ofrecidas por Alexa: la posición en el Ranking Alexa y el número de sitios que enlazan al blog. Estos datos ofrecen una perspectiva interesante en cuanto al posicionamiento de los blogs en relación al resto de webs presentes en Internet. En la tabla 2 se pueden observar algunos estadísticos sobre ambas variables.

\section{Tabla 2}

Variables Alexa

\begin{tabular}{lcccc}
\hline \multicolumn{1}{c}{ Variables Alexa } & $\overline{\mathbf{x}}$ & Desviación típica & Mínimo & Máximo \\
\hline $\begin{array}{l}\text { Posición en Ranking } \\
\text { Alexa }\end{array}$ & 4974891.54 & 4774863.91 & 17839 & 22835163 \\
\hline Sitios que enlazan al blog & 73.62 & 163.52 & 1 & 1215 \\
\hline
\end{tabular}

Fuente: Elaboración propia

Se aprecia la posición media tan baja en el ranking Alexa, lo que implica que los blogs analizados tienen menor tráfico en la red. La desviación típica también ofrece un valor muy alto, indicativo de la amplia variación de las posiciones de los blogs en el ranking. El otro valor, correspondiente al número de sitios webs que enlazan a cada uno de los blogs, ofrece una media de 73.62 enlaces. En un análisis de estas variables, según la formación de los blogueros, podemos encontrar algunas diferencias significativas, como se representa en la tabla 3.

De nuevo, son los blogs cuyos autores cuentan con una formación inicial relacionada con la Pedagogía, Psicología y Educación los que resaltan, pero, en este caso, por tener una repercusión en la red menor que el resto de blogs. Además, también son los que tienen una media menor $(\bar{x}=36.92)$ de enlaces al propio blog. Aunque los blogueros con esta formación de base son los que publican con mayor frecuencia, parecen obtener peores resultados a la hora de sobresalir en la red, lo que puede ser indicativo de una falta de formación en cuanto a estrategias de posicionamiento web. 
Los blogs como recurso de la orientación profesional...

Pilar Martínez-Clares et al.

Tabla 3

Posición y variables Alexa según formación inicial del bloguero

\begin{tabular}{lcccc}
\hline & \multicolumn{2}{c}{ Posición ranking Alexa } & \multicolumn{2}{c}{ Sitios que enlazan (Alexa) } \\
\hline \multicolumn{1}{c}{ Formación } & $\bar{x}$ & Desviación típica & $\overline{\mathbf{x}}$ & Desviación típica \\
\hline ADE & 4632101.63 & 3829382.29 & 71.94 & 112.21 \\
\hline Derecho y Ciencias del Trabajo & 4523885.81 & 3577581.68 & 38.23 & 39.67 \\
\hline Pedagogía, Psicología y Educación & 8803223.71 & 6361935.60 & 36.92 & 112.48 \\
\hline Sociología, Filosofía, Publicidad & 4102886.14 & 3219573.12 & 58.00 & 98.23 \\
\hline Titulaciones técnicas & 2568224.87 & 1865131.47 & 46.67 & 22.23 \\
\hline \multicolumn{1}{c}{$\boldsymbol{x}^{2}(\mathbf{g l})$ Sig. } & \multicolumn{2}{c}{$15.478(4) .009$} & \multicolumn{2}{c}{$14.240(4) .014$} \\
\hline
\end{tabular}

Fuente: Elaboración propia

Objetivo 2. Identificar la comunidad virtual generada en torno a dichos blogs, a través de diferentes tasas de interacción formuladas a partir de los debates iniciados con los contenidos más difundidos.

Uno de los elementos clave en la realidad 2.0 es la comunidad, el conjunto de personas que interactúa en torno a un tema, una red, un blog o un conjunto de éstos. Para la medición de la comunidad, se toma el número de seguidores del bloguero o del blog en las principales redes sociales (Linkedln, Facebook y Twitter). La prueba de Friedman señala la existencia de diferencias significativas en el número de seguidores según la red social de que se trate. Los estadísticos se muestran en la tabla 4.

\section{Tabla 4}

Seguidores del bloguero en las principales redes sociales. Prueba de Friedman

\begin{tabular}{|c|c|c|c|c|}
\hline Redes sociales & $\overline{\mathbf{x}}$ & $\begin{array}{l}\text { Desviación } \\
\text { típica }\end{array}$ & $\begin{array}{c}\text { Rango } \\
\text { promedio }\end{array}$ & Prueba de Friedman \\
\hline Linkedln & 3331.11 & 6804.79 & 1.85 & \multirow{3}{*}{$\begin{array}{c}x^{2}=45.970 ; g l=2 ; \\
p=.000\end{array}$} \\
\hline Facebook & 40026.65 & 268372.34 & 1.51 & \\
\hline Twitter & 23768.07 & 66682.82 & 2.64 & \\
\hline Perfiles a los que sigue en Twitter & 2850.20 & 5280.00 & & \\
\hline Comunidad & 86087.00 & 326160.19 & & \\
\hline
\end{tabular}

Fuente: Elaboración propia

En términos generales, se aprecia una importante amplitud del número de seguidores en cada una de las redes sociales, siendo Facebook quien tiene un impacto mayor $(\bar{x}=40026.65$ seguidores), mientras que Linkedln, una red social algo más específica y restringida, aporta una media de 3331.11 seguidores a los blogs. En términos globales, se encuentra un tamaño medio de la comunidad en los blogs analizados de 86087 personas. La prueba $U$ de Mann Whitney señala diferencias en el tamaño de la comunidad ( $p \leq .05)$, según la tipología del blog, sólo en el caso del número de seguidores de Facebook (tabla 5). En este sentido, son los blogs de carácter institucional los que tienen una comunidad mayor en esta red social ( $\bar{x}=120,877.81)$, lo que lleva a pensar que, siendo conocedores de su impacto, implementan más estrategias que les llevan a obtener mejores 
resultados, en este caso, alcanzar un mayor número de seguidores y ampliar su comunidad a través de otro medio, más allá del propio blog.

\section{Tabla 5}

Diferencias en el tamaño de la comunidad por tipo de blog. Prueba $U$ de Mann Whitney

\begin{tabular}{lccccc}
\multicolumn{1}{c}{ Tipos de blog } & $\overline{\mathbf{x}}$ & Desviación típica & Rango promedio & $\begin{array}{c}\text { U de Mann- } \\
\text { Whitney (Sig.) }\end{array}$ \\
\cline { 1 - 4 } Personal & $4,397.32$ & $11,381.36$ & 39.19 & & $\begin{array}{c}542.500 \\
\text { Institucional }\end{array}$ \\
\cline { 1 - 4 } & $120,877.81$ & $481,565.02$ & 51.63 & \\
\hline
\end{tabular}

Fuente: Elaboración propia

Por su parte la prueba $\mathrm{H}$ de Kruskall Wallis no muestra diferencias significativas $(p \leq .05)$ en el tamaño de la comunidad de los blogs según la ocupación de los blogueros, ni en relación a su formación de grado. Tampoco se encuentran diferencias significativas $(p \leq .05)$ en relación a la formación de postgrado, relacionada con Recursos Humanos o Coaching.

Más allá de conocer el tamaño y composición de la comunidad de un blog, otro elemento en el que es necesario profundizar es la interacción que se produce en torno a los diferentes contenidos publicados. Para ello se emplean las tasas de interacción, en las que se relaciona el número de interacciones surgidas a partir de cada uno de los contenidos analizados con el tamaño de la comunidad del blog. En la tabla 6 se presentan los resultados de la prueba de Friedman comparando las diferentes tasas, que señalan que existen diferencias significativas.

Tabla 6

Tasas de interacción. Prueba de Friedman

\begin{tabular}{|c|c|c|c|c|}
\hline Tasas de interacción & $\overline{\mathbf{x}}$ & $\begin{array}{l}\text { Desv. } \\
\text { típica }\end{array}$ & $\begin{array}{c}\text { Rango } \\
\text { promedio }\end{array}$ & $x^{2}(g l)$ Sig. \\
\hline Tasa de interacción comentarios blog & .0095 & .04975 & 1.94 & \multirow{4}{*}{$\begin{array}{c}1752.651 \\
(3) \\
.000\end{array}$} \\
\hline Tasa de interacción Facebook & .1759 & .50357 & 3.04 & \\
\hline Tasa de interacción Linkedln & .3321 & 1.10175 & 3.20 & \\
\hline Tasa de interacción Twitter & .0010 & .01656 & 1.82 & \\
\hline Tasa de interacción total & .5185 & 1.46056 & & \\
\hline
\end{tabular}

Fuente: Elaboración propia

Como se puede observar (tabla 6), existe una mayor interacción a través de Linkedln que a través del resto de herramientas, incluso, más que a través de los comentarios de los propios blogs. Esta información contrasta con el hecho de que Facebook es la herramienta en la que mayor número de personas siguen a los blogueros.

En un análisis de las correlaciones entre las diferentes tasas planteadas (tabla 7) se aprecia que, pese a que la correlación entre las tasas parciales es positiva y significativa $\left({ }^{*} p \leq .05\right)$, los 
coeficientes son moderados en los tres casos, exceptuando la tasa de interacción en Twitter. Esto parece mostrar que determinados contenidos tienen mayor probabilidad de ser compartidos 0 comentados, pero puede aparecer una cierta especialización en torno a las redes sociales dependiendo del blog o el contenido compartido.

\section{Tabla 7}

Correlaciones relacionadas con visibilidad, actividad e interacción. Prueba de Spearman
Tasas de interacción
(1)
(2)
(3)
(4)
(5)

\begin{tabular}{|c|c|c|c|c|}
\hline (1) Tasa de interacción global & 1 & & & \\
\hline (2) Tasa de interacción comentarios blog &, $467^{*}$ & 1 & & \\
\hline (3) Tasa de interacción Facebook & ,766* &, $434^{*}$ & 1 & \\
\hline (4) Tasa de interacción Linkedln & $954^{*}$ & ,422* &, $565^{*}$ & 1 \\
\hline (5) Tasa de interacción Twitter & 045 & 025 & ,033 & 031 \\
\hline
\end{tabular}

Fuente: Elaboración propia

Igualmente, en la tabla 8 se exponen los resultados del análisis comparativo según la ocupación del bloguero y su formación, apreciándose diferencias significativas en todos los casos. Esto muestra que el perfil del orientador, tanto en relación a la ocupación como a su formación, tiene un importante impacto en la interacción y generación de comunidad en torno al blog.

Tabla 8

Tasas de interacción según ocupación y formación de Grado del bloguero. Prueba H de Kruskal-Wallis

\begin{tabular}{|c|c|c|c|c|c|c|c|c|c|c|}
\hline \multirow[b]{2}{*}{ Ocupación } & \multicolumn{2}{|c|}{$\begin{array}{c}\text { Comentarios } \\
\text { Blog }\end{array}$} & \multicolumn{2}{|c|}{ Facebook } & \multicolumn{2}{|c|}{ Linkedln } & \multicolumn{2}{|c|}{ Twitter } & \multicolumn{2}{|c|}{ Global } \\
\hline & $\overline{\mathrm{X}}$ & D.T. & $\overline{\mathrm{x}}$ & D.T. & $\bar{x}$ & D.T. & $\overline{\mathrm{x}}$ & D.T. & $\overline{\mathrm{x}}$ & D.T. \\
\hline $\begin{array}{l}\text { Directores } \\
\text { y gerentes }\end{array}$ & .007 & .021 & .316 & .408 & .682 & 1.187 & .000 & .000 & 1.484 & 1.550 \\
\hline $\begin{array}{l}\text { Consultor / } \\
\text { Técnico } \\
\text { RRHH }\end{array}$ & .029 & .092 & .514 & .868 & 1.454 & 2.623 & .000 & .000 & 2.277 & 3.548 \\
\hline $\begin{array}{l}\text { Formador / } \\
\text { docente }\end{array}$ & .036 & .063 & .367 & .408 & .967 & 1.321 & .000 & .000 & 2.608 & 1.598 \\
\hline Orientador & .002 & .018 & .057 & .270 & .101 & .421 & .000 & .000 & .222 & .600 \\
\hline Otros & .013 & .069 & .051 & .288 & .052 & .198 & .023 & .076 & .793 & .811 \\
\hline$x^{2}$ (gl) Sig. & & $\begin{array}{l}7.739 \\
.000\end{array}$ & & $\begin{array}{l}.651 \\
.000\end{array}$ & & $\begin{array}{l}649 \\
000\end{array}$ & & $\begin{array}{l}136 \\
000\end{array}$ & $(4$ & \\
\hline
\end{tabular}

Fuente: Elaboración propia 
Los blogs como recurso de la orientación profesional...

Pilar Martínez-Clares et al.

Tabla 8 (Continuación)

Tasas de interacción según ocupación y formación de Grado del bloguero. Prueba $\mathrm{H}$ de Kruskal-Wallis

\begin{tabular}{|c|c|c|c|c|c|c|c|c|c|c|}
\hline \multirow[b]{2}{*}{ Formación } & \multicolumn{2}{|c|}{$\begin{array}{c}\text { Comentarios } \\
\text { Blog }\end{array}$} & \multicolumn{2}{|c|}{ Facebook } & \multicolumn{2}{|c|}{ Linkedln } & \multicolumn{2}{|c|}{ Twitter } & \multicolumn{2}{|c|}{ Global } \\
\hline & $\overline{\mathrm{x}}$ & D.T. & $\bar{x}$ & D.T. & $\bar{x}$ & D.T. & $\bar{x}$ & D.T. & $\bar{x}$ & D.T. \\
\hline ADE & .003 & .011 & .151 & .283 & .473 & .651 & .000 & .000 & .845 & .957 \\
\hline $\begin{array}{c}\text { Derecho y } \\
\text { Ciencias del } \\
\text { Trabajo }\end{array}$ & .005 & .029 & .167 & .435 & .174 & .479 & .000 & .000 & .435 & .827 \\
\hline $\begin{array}{l}\text { Pedagogía, } \\
\text { Psicología y } \\
\text { Educación }\end{array}$ & .027 & .064 & .310 & .736 & .975 & 1.758 & .000 & .000 & 1.213 & 2.034 \\
\hline $\begin{array}{l}\text { Sociología, } \\
\text { Filosofía, } \\
\text { Publicidad }\end{array}$ & .035 & .093 & .681 & 1.264 & 3.631 & 3.337 & .000 & .000 & 7.342 & 4.819 \\
\hline $\begin{array}{l}\text { Titulaciones } \\
\text { técnicas }\end{array}$ & .056 & .114 & .371 & .615 & .704 & 1.013 & .013 & .057 & 1.489 & 1.521 \\
\hline$x^{2}$ (gl) Sig. & $\begin{array}{l}195 \\
(4)\end{array}$ & & $\begin{array}{l}37 \\
(4)\end{array}$ & & $\begin{array}{l}94 \\
(4)\end{array}$ & & & & $\begin{array}{l}91 . \\
(4)\end{array}$ & $\begin{array}{l}20 \\
000\end{array}$ \\
\hline
\end{tabular}

Fuente: Elaboración propia

Otro elemento de interés a tener en cuenta es la diferente interacción que puede provocar cada tipología de contenidos publicados. La tabla 9 recoge la tasa de interacción total para cada tipo de contenido publicado.

Dadas las diferencias en las tasas de interacción, se pone de relieve la importancia del contenido abordado en las publicaciones a la hora de generar interacción entre el blog y sus lectores. De la misma manera, es posible conocer los intereses de la comunidad, lo que puede ayudar al bloguero a enfocar sus entradas o posts hacia la satisfacción de las necesidades de sus usuarios.

Tabla 9

Tasa de interacción por categorías de contenido

\begin{tabular}{lcc}
\hline \multicolumn{1}{c}{ Contenidos } & $\overline{\mathbf{X}}$ & D.T. \\
\hline Competencias transversales & 1.539 & 2.576 \\
\hline Redes sociales & 1.518 & 2.546 \\
\hline Búsqueda activa de empleo & 1.367 & 2.750 \\
\hline Desarrollo de la carrera profesional & 1.295 & 2.046 \\
\hline Gestión de recursos humanos & 1.253 & 1.772 \\
\hline Off-topic & 1.128 & 1.328 \\
\hline Coaching & 1.082 & .811 \\
\hline Marca personal & 0.297 & .686 \\
\hline Derecho laboral & 0.283 & .412 \\
\hline
\end{tabular}

Fuente: Elaboración propia 
Tabla 9 (Continuación)

Tasa de interacción por categorías de contenido

\begin{tabular}{lcc}
\hline \multicolumn{1}{c}{ Contenidos } & $\overline{\mathbf{x}}$ & D.T. \\
\hline Formación & 0.257 & .588 \\
\hline Emprendimiento & 0.235 & .690 \\
\hline Meta-blog & 0.235 & .268 \\
\hline Mercado laboral & 0.216 & .713 \\
\hline Profesional de la orientación & 0.209 & .363 \\
\hline Trabajo en el extranjero & 0.166 & .203 \\
\hline Becas & 0.147 & .496 \\
\hline Eventos & 0.102 & .147 \\
\hline Ofertas de empleo & 0.099 & .218 \\
\hline Cualificación y acreditación & 0 & 0
\end{tabular}

Fuente: Elaboración propia

Finalmente, se aborda un análisis de los debates que se generan en los comentarios del propio blog, con el objeto de valorar el modo en que se discute, debate e intercambian opiniones. Para ello se establece una categoría de debates según su nivel de profundidad, diferenciando entre debates simples y debates puros o complejos (tabla 10).

\section{Tabla 10}

Tipología de debates en comentarios (\%)

\begin{tabular}{|c|c|c|c|c|c|}
\hline \multirow[t]{3}{*}{ Tipos de blog } & \multicolumn{5}{|c|}{ Tipos de debate } \\
\hline & \multirow[t]{2}{*}{ Sin debate } & \multicolumn{3}{|c|}{ Debate simple } & \multirow{2}{*}{$\begin{array}{l}\text { Debate puro } \\
\text { complejo }\end{array}$} \\
\hline & & $\begin{array}{c}\text { Baja } \\
\text { participación }\end{array}$ & $\begin{array}{c}\text { Tipo de } \\
\text { interacción }\end{array}$ & $\begin{array}{l}\text { Tipo de } \\
\text { contenido }\end{array}$ & \\
\hline Personal & 82.26 & 12.79 & 0.68 & 1.21 & 3.06 \\
\hline Institucional & 90.72 & 5.57 & 1.86 & 0.78 & 1.08 \\
\hline Total & 85.56 & 9.97 & 1.13 & 1.04 & 2.29 \\
\hline
\end{tabular}

Fuente: Elaboración propia

Se aprecia que un porcentaje muy elevado de publicaciones no llegan a generar ningún tipo de conversación (85.56\%), además sólo un porcentaje muy pequeño llega a producir un verdadero debate $(2.29 \%)$ El resto quedan incluidos en lo que se ha denominado debates simples. En la comparación a través de la prueba $X^{2}$ se comprueba que hay diferencias significativas $\left(x^{2}=93.930\right.$; $\mathrm{gl}=4 ; p=.000$ ) en cómo los blogs de carácter institucional o de carácter personal son capaces de generar debates en el contenido que publican. Aunque el porcentaje es muy bajo, son los blogs personales los que generan un mayor número de debates puros o complejos (3.06\%), en los que verdaderamente se produce un proceso de comunicación, existe intercambio y puede producirse un mayor aprendizaje. Tal vez esto sea posible por el carácter más cercano y directo con el que el bloguero interacciona con su comunidad. Los blogs institucionales, debido a ese carácter 
corporativo y de negocio, interesan más a una gran comunidad, pues su fin es mantener informada a ésta a través de publicitar sus productos y/o servicios.

\section{Conclusiones}

En el análisis de los blogs de orientación profesional es necesario tener en cuenta diversas variables y realidades, tanto referidas a la visibilidad de sus contenidos y frecuencia de publicación como a la vinculación que se produce entre los blogs y su audiencia. En este trabajo se halla una alta variabilidad en la frecuencia de publicación, tanto por las diferencias entre los blogs personales o institucionales como por la alta desviación típica de la frecuencia en ambas tipologías de blogs. Esta dispersión da cuenta de la compleja realidad de la blogosfera de orientación profesional, en la que intervienen un numeroso grupo de agentes, profesionales e instituciones, con una amplia variedad formativa de base, ocupación y concepciones sobre los procesos y objetivos de la orientación, que es necesario conocer y abordar para poder mejorar la integración de la web 2.0 en dichos procesos.

La intensidad de publicación de los blogueros puede estar determinada por algunos factores como el propio carácter del blog o la ocupación profesional y formación de su autor. Las diferencias encontradas según la ocupación y formación de los blogueros permiten entender mejor la realidad y motivaciones de los mismos a la hora de mantener activo un blog, así como el impacto que esto tiene en la visibilidad del mismo y en la vinculación con los seguidores.

Estrechamente unido a la intensidad de publicación, la visibilidad de los blogs es clave para cualquier creador de contenidos en la red (Kumar, Ande, Kumar y Singh, 2018), por ello este estudio presenta un análisis de dos variables: la posición en el ranking Alexa y el número de sitios que enlazan al blog, obtenido a partir de la propia herramienta Alexa. Los blogs analizados presentan una posición Alexa muy baja, aunque también con una dispersión muy importante, siendo la posición más alta la no 17839 [las primeras posiciones corresponden a las webs de Google $\left(1^{\circ}\right)$,

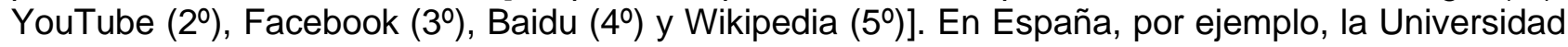
de Murcia ocupa la posición no 13795, mientras que la web de la Universidad Complutense de Madrid ocupa el no 7838.

En el análisis sobre la comunidad generada en torno a los blogs se estudia el número de personas o perfiles en redes sociales que siguen al blog o al responsable del blog en tres redes sociales. Los hallazgos encontrados permiten concluir que los blogs tienen comunidades muy amplias de seguidores, aunque con alto grado de dispersión. El tamaño de estas comunidades puede indicar el grado de compromiso o vinculación de la audiencia con el blog, hecho que diferentes estudios (Aguilera y Baños, 2017; Magno, 2017; Vepsäläinen, Li y Suomi, 2017) relacionan directamente con el impacto de los blogs en sus usuarios y en sus decisiones.

En un análisis más pormenorizado de las comunidades de seguidores, destaca el tamaño medio de los seguidores en Facebook frente a Twitter y muy lejos de Linkedln, lo que coincide con los resultados del Estudio Anual de Redes Sociales, llevado a cabo en España por IAB Spain (2019), en el que Facebook es la segunda red social más utilizada por los usuarios de Internet; en una quinta posición se encuentra Twitter y en sexto lugar Linkedln. Además, aparecen diferencias en el número de seguidores según la tipología del blog, siendo los de carácter personal mucho más pequeños en cuanto a su comunidad que los de carácter institucional. Estos datos parecen ser el reflejo de distintos niveles de especialización de las redes sociales, así como de la facilidad de determinados blogs institucionales para captar seguidores. 
Se presenta también un análisis de las interacciones que se producen en torno a dicha comunidad, la cual ofrece una perspectiva muy interesante en cuanto a reacciones de la comunidad ante las publicaciones de los blogs. Para ello se emplean las tasas de interacción, que ponen en relación el número de interacciones medias de cada publicación con el tamaño de la red o comunidad en torno a dicho blog. Esto ofrece una visión sobre la cantidad de interacciones (número de veces que se comparte en redes sociales o número de comentarios realizados) relativas al tamaño de la comunidad.

En esta investigación, es Linkedln donde se halla una tasa de interacción mayor, siendo Twitter la que menos interactúa, lo que parece contradecir la percepción popular. En todo caso, en los datos combinados de las diferentes redes sociales destacan la importancia de las estrategias de multicanalidad por parte de los blogueros, aprovechando el impacto, importancia y diferentes realidades de las redes sociales disponibles. Esto se corrobora a partir del análisis de correlaciones entre las tasas de interacción parciales, que resultan ser positivas, pero con un carácter moderado, lo que parece indicar que determinados posts reciben mayor interacción, aunque ésta no es lineal, sino que depende del tipo de contenido, autor, etc. Pueden provocar diferentes niveles de interacción en diferentes redes sociales.

Los análisis realizados muestran que las tasas de interacción quedan influenciadas por diferentes variables, tanto relacionadas con el propio bloguero (ocupación y formación) como con el propio tipo de contenido publicado. Se concluye que los contenidos de tipo informativo parecen generar una menor interacción, mientras que otros contenidos parecen ser más susceptibles de debate o comentario, conclusión coincidente con otros estudios (Kim \& Yang, 2017; Kulkarni et al., 2013) que señalan que determinadas variables o características de los contenidos publicados tienen un impacto en el modo en que los usuarios se involucran con el mismo, comentándolo, compartiendo en redes sociales, etc.

En el análisis realizado en torno a los debates generados destaca que sólo un pequeño porcentaje de los contenidos llega a generar un debate considerado puro o complejo, lo que lleva a la necesidad de reflexionar sobre la importancia de generar una interacción de mayor calidad en relación a los contenidos publicados en los blogs, especialmente, valorando lo señalado por Top (2012) y Bernal (2015), la relación entre la interacción producida en torno a los blogs y el aprendizaje que éstos generan. Este análisis se sitúa en la línea de otros estudios que comienzan a profundizar en estrategias y estilos comunicativos; por ejemplo, Luzón (2013) analiza el conflicto y la discusión en algunos blogs, Zell y Moeller (2015) estudian y subrayan la importancia de la interacción para valorar los propios contenidos, mientras que Busso (2017) analiza diferentes reacciones agresivas o exaltadas, diferenciándolas por su finalidad disruptiva (trolling) o por la mera exaltación verbal, sin intención de ruptura del diálogo (flaming).

Las posibilidades de las redes sociales y la web 2.0 en la orientación profesional no se centran exclusivamente en la interconectividad, sino en la interactividad: cómo las personas conversan, colaboran, cooperan y aprenden. No es suficiente una conexión sin una interacción que genere aprendizaje. Como destacan Yang y Kang (2009), la interacción de los usuarios con los blogs va a quedar influenciada por su vinculación con el mismo, los contenidos que publica, así como por la percepción de cercanía o comodidad para interactuar con el autor, variables consistentes con los resultados de esta investigación. Este tipo de elementos señalan algunas condiciones relevantes a la hora de establecer un proceso de orientación: vinculación, compromiso, interacción o confianza.

Las herramientas TIC, especialmente la web 2.0, pueden convertirse en un importante aliado de la orientación profesional, pero es necesario ir más allá de un empleo simple de las mismas y potenciar todas aquellas estrategias que permitan construir una base sólida desde la que trabajar la orientación profesional. Así podrá convertirse verdaderamente en ese proceso continuado en el que los orientados adquieren un verdadero protagonismo, participando de un modo activo en la construcción del conocimiento y en el desarrollo de competencias para la inserción sociolaboral. 
Finalmente, como prospectiva, es conveniente continuar indagando y profundizando en las posibilidades orientadoras de los blogs, con la finalidad de optimizar el proceso orientador, complementándolo de forma on-line con calidad. Para contribuir a esto será preciso el estudio de nuevas dimensiones e indicadores que garanticen esa calidad en cuanto a la información, contenidos, servicios, herramientas, interacción, comunicación... proporcionados desde la blogosfera de la orientación profesional. También sería interesante ampliar el estudio a otras redes sociales (directas) y páginas web. De esta manera, podría darse respuesta a la doble pregunta que da origen a esa investigación más amplia de la que forma parte este trabajo: qué aporta la Web 2.0 a la orientación profesional y cómo integrar esas posibilidades de manera útil en el proceso orientador.

La respuesta a esta cuestión no es sencilla, ya que es preciso tener en cuenta la mayor limitación de este trabajo: cómo abordar un espacio sin límites, tanto en lo referido a la blogosfera como a la red de redes, Internet. Un espacio que, además, está en continua actualización, por lo que nunca se termina de concluir si todo está representado. De ahí que también sea preciso un continuo seguimiento de su evolución, con la intención de aprovechar todas las virtualidades de la Web 2.0 en el proceso orientador, pues no podemos obviar que cada vez son más los usuarios de Internet los que acceden a la red con fines profesionales y para buscar empleo (IAB Spain, 2019), por lo que no puede descuidarse este nuevo contexto de la orientación.

\section{Referencias bibliográficas}

Aguilera, J. y Baños, M. (2017). Las comunicaciones en el nuevo paradigma de marketing. Experiencias, relevancia, engagement y personalización. ICONO14, Revista Científica de Comunicación y Tecnologías emergentes, 15(2), 1-15. https://doi.org/10.7195/ri14.v15i2. 1098

Barraza, A. (2013). Presencia discursiva del estrés académico en la blogósfera. Un análisis de contenido de treinta blogs. Revista Iberoamericana de Psicología: Ciencia y Tecnología, 6(2), 45-54.

Busso, M.P. (2017). Los foros de Internet como espacios de la migración mediatizada de argentinos. ICONO14, Revista Científica de Comunicación y Tecnologías emergentes, 15(1), 23-44. https://doi.org/10.7195/ri14.v15i1.983

Bernal, R.M. (2015). Herramientas telemáticas para la comunicación educativa: catalogación, análisis y posibilidades de uso de los blogs. https://digitum.um.es/digitum/handle/10201/ 45695

Castells, M. (2012). La educación en la sociedad en red. En C. Jiménez, J.L. García, B. Álvarez y J. Quintanal (Eds.), Investigación y educación en un mundo en red (pp. 1-6). McGraw Hill.

Cogoi, C. (2002). Videoconferencia y orientación. Ámbitos de aplicación y ejemplos de buenas prácticas. Revista Española de Orientación y Psicopedagogía, 13(1), 5-15.

Cuevas, V.J. (2011). El blog como herramienta de orientación académica. En J. Martín y A. Cobos (Coord.), V Encuentro Nacional de Orientación: Educar y orientar en la diversidad (pp. 299308). COPOE. 
De Pablos, E. (2016). Revolución 4.0. CyC Prisma, 21, 10-15.

Del Fresno, M., Daly, A.J. y Segado, S. (2016). Identificando a los nuevos influyentes en tiempos de Internet: medios sociales y análisis de medios sociales. Revista Española de Investigaciones Sociológicas, 153, 23-42. https://doi.org/10.5477/cis/reis.153.23

Echeverría, B. y Martínez, P. (2018). Revolución 4.0, competencias, educación y orientación. RIDU: Revista Digital de Investigación en Docencia Universitaria, 12(2), 4-34. https://doi.org/10.19083/ridu.2018.831

Fernández González, M. (2015). Orientación profesional para el empleo en la web 2.0 y preparándonos para la 3.0. Comisiones Obreras de Extremadura.

Fuchs, C. (2011). Teaching and learning. Guide for new media, web 2.0 and surveillance. Sociology Compass, 5(2), 134-147.

Fumero, A. (2005). Un tutorial sobre blogs. El abecé del universo blog. Telos: Cuadernos de Comunicación e Innovación, 65, 46-59.

Gómez Cruz, E. (2002). Espacio, ciberespacio e hiperespacio: Nuevas configuraciones para leer la comunicación mediada por computadora. En F.J. Aceves (Ed.), Anuario de investigación de la comunicación CONEICC (pp. 271-285). CONEICC.

González-Benito, A. y Otero, A. (2019). Las TIC aplicadas en el desarrollo de la acción tutorial y orientadora. En REDINE (Ed.), Conference Proceedings EDUNOVATIC 2018 (pp. 254-255). Adaya Press.

Hernández, D., Ramírez-Martinell, A. y Cassany, D. (2014). Categorizando a los usuarios de sistemas digitales. Píxel-Bit. Revista de Medios y Educación, 44(1), 113-126. https://doi.org/10.12795/pixelbit.2014.i44.08

Hernández, R., Fernández, C. y Baptista, P. (2014). Metodología de la investigación (6ª ed.). McGraw-Hill Education.

IAB Spain (2019). Estudio anual de redes sociales 2019. https://iabspain.es/estudio/estudio-anualde-redes-sociales-2019/

Kim, C. y Yang, S.U. (2017). Like, comment, and share on Facebook: How each behavior differs from the other. Public Relations Review, 43(2), 441-449. https://doi.org/10.1016/j.pubrev. 2017.02.006

Kulkarni, A., Joseph, S., Raman, R., Bharathi, V., Goswami, A. y Kelkar, B. (2013). Blog content and user engagement. An insight using statistical analysis. International Journal of Engineering and Technology, 5(3), 2719-2733. https://papers.ssrn.com/sol3/papers.cfm? abstract_id=2294267

Kumar, N., Ande, G., Kumar, J.S. y Singh, M. (2018). Toward maximizing the visibility of content in social media brand pages: a temporal analysis. Social Network Analysis and Mining, 8(1), 114. https://doi.org/10.1007/s13278-018-0488-z

Luzón, M.J. (2013). This is an erroneous argument: Conflict in academic blog discussions. Discourse, Context and Media, 2, 111-119. https://doi.org/10.1016/j.dcm.2013.04.005

Magno, F. (2017). The influence of cultural blogs on their readers' cultural product choices, International Journal of Information Management, 37(3), 142-149. https://doi.org/10.1016/ j.ijinfomgt.2017.01.007 
Los blogs como recurso de la orientación profesional...

Pilar Martínez-Clares et al.

Martínez Clares, P. (Coord.) (2017). Manual de Orientación Educativa y Profesional. DM.

Martínez, M., González, N. y Pérez, J. (2018). Aproximación al perfil formativo del orientador profesional en la blogosfera. Revista de Investigación Educativa, 36(1), 39-56. https://doi.org/10.6018/rie.36.1.306401

Manzanares, A. y Sanz, C. (2018). El modelo de orientación profesional en cuestión. Revisión de sus referentes teórico-prácticos. En A. Manzanares y C. Sanz (Eds.), Orientación profesional. Fundamentos y estrategias (pp.65-85). Ediciones de la Universidad de CastillaLa Mancha

Muñoz-Carril, P.C. y González-Sanmamed, M. (2014). Posibilidades de la web 2.0 en Orientación Educativa: un estudio exploratorio sobre su presencia en las webs de los Departamentos de Orientación de Secundaria. Revista Española de Orientación y Psicopedagogía, 25(3), 3655. https://doi.org/10.5944/reop.vol.25.num.3.2014.13857

Net Market Share (s.f.). Search engine market share. NET MARKETSHARE. https://bit.ly/ 2WCEAxq

Observatorio Nacional de las Telecomunicaciones y de la Sociedad de la Información (2011). Las Redes Sociales en Internet. https://www.ontsi.red.es/ontsi/sites/ontsi/files/redes_socialesdocumento_0.pdf

Orihuela, J.L. (2006). La revolución de los blogs. La Esfera de los Libros.

Piñeiro, G. (2019). La realidad de la web 3.0. Harvard Deusto Marketing y Ventas, 153, 6-11.

Romero, C. y Montilla, M. (2015). La utilización de las TIC en la orientación educativa: un estudio exploratorio sobre la situación actual de uso y formación entre los profesionales de la orientación. Revista Española de Orientación y Psicopedagogía, 26(3), 78-95. https://doi.org/10.5944/reop.vol.26.num.3.2015.16402

Sáez Vacas, F. (2005). La blogosfera: un vigoroso subespacio de comunicación en Internet. Telos: Cuadernos de Comunicación e Innovación, 64, 14-17.

Schwab, K. (2016, 14 de junio). The Fourth Industrial Revolution: what it means, how to respond. World Economic Forum. https://www.weforum.org/agenda/2016/01/the-fourth-industrialrevolution-what-it-means-and-how-to-respond/

Sobrado, L.M., Ceinos, C. y García, R. (2012). Utilización de las TIC en orientación profesional: experiencias innovadoras. Revista Mexicana de Orientación Educativa, 9(23), 2-10.

Suárez, J.M., Pérez, S., Boza A. y García-Valcárcel, A. (2012). Educación 2.0. Horizontes de la innovación en educación. En C. Jiménez, J.L. García, B. Álvarez y J. Quintanal (Eds.), Investigación y educación en un mundo en red (pp. 1-6). McGraw Hill.

Top, E. (2012). Blogging as a social medium in undergraduate courses: sense of community best predictor of perceived learning. Internet and Higher Education, 15(1), 24-28. https://doi.org/ 10.1016/j.iheduc.2011.02.001

Veiga, D., Valverde, M.A., Castilla, C. y Diz, M. (2017). La aportación de los blogs a la orientación educativa en red. Revista AOSMA, 23, 36-47.

Vepsäläinen, T., Li, H. y Suomi, R. (2017). Facebook likes and public opinion: Predicting the 2015 Finnish parliamentary elections. Government Information Quarterly, 34(3), 524-532. https://doi.org/10.1016/j.giq.2017.05.004 
Yang, S. U. y Kang, M. (2009). Measuring blog engagement: Testing a four-dimensional scale. Public Relations Review, 35(3), 323-324. https://doi.org/10.1016/j.pubrev.2009.05.004

Zell, A. L. y Moeller, L. (2018). Are you happy for me... on Facebook? The potential importance of "likes" and comments. Computers in Human Behavior, 78, 26-33. https://doi.org/10.1016/ j.chb.2017.08.050

Fecha de entrada: 17 septiembre 2019

Fecha de revisión: 6 mayo 2020

Fecha de aceptación: 21 mayo 2020 